\title{
Reduced Neuronal Inhibition and Coordination of Adolescent Prefrontal Cortex during Motivated Behavior
}

\author{
David A. Sturman and Bita Moghaddam \\ Department of Neuroscience, University of Pittsburgh, Pittsburgh, Pennsylvania 15260
}

\begin{abstract}
Adolescence is a time of both cognitive maturation and vulnerability to several major psychiatric illnesses and drug dependence. There is increasing awareness that behavioral or pharmacological intervention during this period may be critical for disease prevention in susceptible individuals. Therefore, we must attain a deeper understanding of how the adolescent brain processes salient events relevant to motivated behavior. To do this, we recorded single-unit and local field potential activity in the orbitofrontal cortex of rats as they performed a simple reward-driven operant task. Adolescents encoded basic elements of the task differently than adults, indicating that neuronal processing of salient events differs in the two age groups. Entrainment of local field potential oscillations, variance in spike timing, and relative proportions of inhibitory and excitatory responses differed in an event-specific manner. Overall adolescent phasic neural activity was less inhibited and more variable through much of the task. Cortical inhibition is essential for efficient communication between neuronal groups, and reduced inhibitory control of cortical activity has been implicated in schizophrenia and other disorders. Thus, these results suggest that reduced inhibitory responses of adolescent cortical neurons to salient events could be a critical mechanism for some of the increased vulnerabilities of this period.
\end{abstract}

\section{Introduction}

Adolescence is a time of adjustment as one completes the physical and psychosocial transitions to adulthood (Arnett, 1999). It is also considered a period of vulnerability as it coincides with the onset of symptoms for several major psychiatric problems, including mood disorders, schizophrenia, and drug abuse (Volkmar, 1996; Pine, 2002; Johnston et al., 2008). In recent years, studies in adolescent humans and animal models have described age-related shifts in cellular and molecular brain architecture and disparities in the pharmacological effects of various drugs (Spear and Brake, 1983; Spear, 2000; Adriani et al., 2004; Brenhouse et al., 2008; Paus, 2010). Age-related behavioral differences have also been examined and are often focused on, although adolescent behavior tends to be quite similar to that of adults in most contexts with only modest changes in decision-making capacity from mid-adolescence onward (Spear, 2000; Figner et al., 2009; Cauffman et al., 2010). Nevertheless, adolescents may process salient events differently from adults. For example, a recent study observed greater adolescent than adult c-Fos protein expression in dorsal striatum and nucleus accumbens after exposure to a reward-associated odor cue (Friemel et al. 2010). Differences in measures of adolescent prefrontal cortex (PFC) neural activity and connectivity have also been described (Ernst et al., 2006; Galvan et al., 2006; Liston et al., 2006; Uhlhaas et al., 2009a; Geier

Received Aug. 11, 2010; revised Nov. 2, 2010; accepted Nov. 5, 2010.

This work was supported by National Institute of Mental Health, the Pittsburgh Life Sciences Greenhouse, and the Andrew Mellon Foundation for a predoctoral fellowship (D.A.S.). We thank Jesse Wood and Yunbok Kim for insightful discussions and Churchland et al. (2010) for making Matlab variability functions available.

Correspondence should be addressed to Bita Moghaddam, University of Pittsburgh, Department of Neuroscience, A210 Langley Hall, Pittsburgh, PA 15260. E-mail: bita@pitt.edu.

DOI:10.1523/JNEUROSCI.4210-10.2011

Copyright $\odot 2011$ the authors $\quad 0270-6474 / 11 / 311471-08 \$ 15.00 / 0$ et al., 2010). However, little is known of the precise nature of these age-related disparities at the neuronal level.

To directly compare the dynamic processing of cortical neurons in adolescents with that of adults, we recorded single-unit and local field potential (LFP) activity from the orbitofrontal cortex (OFC) of rats as they performed a reward-motivated behavior. The OFC was targeted because of its central role in processing value expectation and previous evidence of its underdevelopment in adolescents (Schultz et al., 2000; Galvan et al., 2006; Schoenbaum et al., 2009). The behavioral task involved acting on a learned actionoutcome association (Sturman et al., 2010), which is a fundamental building block of complex motivated behavior. The simplicity of this task allowed for behavioral measures to be very similar between groups. We could therefore test the hypothesis that, even with similar task performance, the adolescent OFC encodes salient taskrelated information differently that adults. Characterizing such fundamental neural activity differences - and doing so at the neuronal level—is critical for identifying developmental processes that may be associated with the increasing neuropsychiatric risks of adolescence and for the future design of intervention strategies to prevent and treat such problems.

\section{Materials and Methods}

Subjects. Adolescent [postnatal day 28 (P28) to P42; $n=8$ ] and adult (P70 and older; $n=4$ ) male Sprague Dawley rats (Harlan) were used. Juvenile (P21) and adult rats were received 1 week before surgery. Subjects were housed in a climate-controlled vivarium under $12 \mathrm{~h}$ light/dark conditions (lights on at 7:00 P.M.), with ad libitum access to chow and water before training. All animal use procedures were approved by the University of Pittsburgh Animal Care and Use Committee.

Surgery and electrophysiology methods. Rats underwent electrode array implantation surgeries as described previously (Totah et al., 2009). Briefly, microelectrode arrays (NB Labs), consisting of eight Teflon- 
insulated stainless-steel wires arranged in a $2 \times$ 4 pattern, were implanted in the OFC. Adults were implanted bilaterally $2.8-3.8 \mathrm{~mm}$ anterior to bregma, $3.1-3.5 \mathrm{~mm}$ lateral to bregma, and $4.5 \mathrm{~mm}$ ventral to the dura surface. Adolescents (P28-P29) were implanted unilaterally (because of size limitations) $2.8-3.2 \mathrm{~mm}$ anterior to bregma, $2.8-3.2 \mathrm{~mm}$ lateral to bregma, and $4.0 \mathrm{~mm}$ ventral to the dura surface. During recordings, a unity-gain junction field-effect transistor head stage attached to a light-weight cable (NB Labs) was connected to a commutator (NB Labs) that allowed rats to move freely within the testing box. Recorded single-unit activity was amplified at $1000 \times$ gain and analog bandpass filtered at $300-8000$ $\mathrm{Hz}$; LPFs were bandpass filtered at $0.7-170 \mathrm{~Hz}$. Single-unit activity was digitized at $40 \mathrm{kHz}$, and LFPs were digitized at $40 \mathrm{kHz}$ and downsampled to $1 \mathrm{kHz}$ by Recorder software (Plexon). Single-unit activity was digitally high-pass filtered at $300 \mathrm{~Hz}$, and LFPs were low-pass filtered at $125 \mathrm{~Hz}$. Behavioral event markers from the operant box were sent to Recorder to mark events of interest. Single units were isolated in Offline Sorter (Plexon) using a combination of manual and semiautomatic sorting techniques as described previously (Homayoun and Moghaddam, 2008; Totah et al., 2009).

Behavior. Adult and adolescent rats were tested in an operant box apparatus (Coulbourn Instruments) that contained a house light, a pellet magazine that could deliver food pellets (fortified dextrose, $45 \mathrm{mg}$; Bio-Serv) into a food trough, and three nose-poke holes arrayed horizontally on the wall opposite the food trough. After 5-6 d of surgical recovery, animals were mildly food restricted, underwent habituation to the behavioral testing ap-

paratus, and began training on the behavioral task, which has been characterized previously (Sturman et al., 2010). Briefly, rats learned to poke into an illuminated center nose-poke hole for food-pellet reinforcement. Trials began with the onset of a cue light inside the center nosepoke hole. When the rat poked into that hole, the light immediately turned off and a single pellet was delivered to the food trough, which was then illuminated. Poking into the food trough to receive the pellet turned off the food-trough light and triggered a $5 \mathrm{~s}$ intertrial interval (Fig. $1 \mathrm{~A}$ ). Each session was terminated after 100 trials or the passage of $30 \mathrm{~min}$. Previous work demonstrated that this task can be quickly learned by both adolescents and adults, with expected maximal performance by the third day of training (Sturman et al., 2010). Main task-performance measures included the number of total trials completed during each session, the latency from cue to instrumental poke, and the latency from instrumental poke to food-trough entry (pellet retrieval). Age $\times$ session repeatedmeasures ANOVAs were performed on all outcome measures in SPSS $(\alpha=0.05)$. In all cases in which the assumption of sphericity was violated, the lower-bound corrections were used for a maximally conservative degrees-of-freedom adjustment.

Histology. After completion of the experiment, rats were anesthetized with chloral hydrate $(400 \mathrm{mg} / \mathrm{kg}$, i.p.), and a $200 \mu \mathrm{A}$ current was passed through recording electrodes for $5 \mathrm{~s}$ to mark electrode tip placements. Animals were perfused with saline and $10 \%$ buffered formalin. Brains were then removed and placed in $10 \%$ formalin. Brains were sectioned in coronal slices, stained with cresyl violet, and mounted to microscope slides. Electrode-tip placements were examined under a light microscope. Only rats with correct placements within the OFC (Fig. $1 B$ ) were included in electrophysiological analyses.
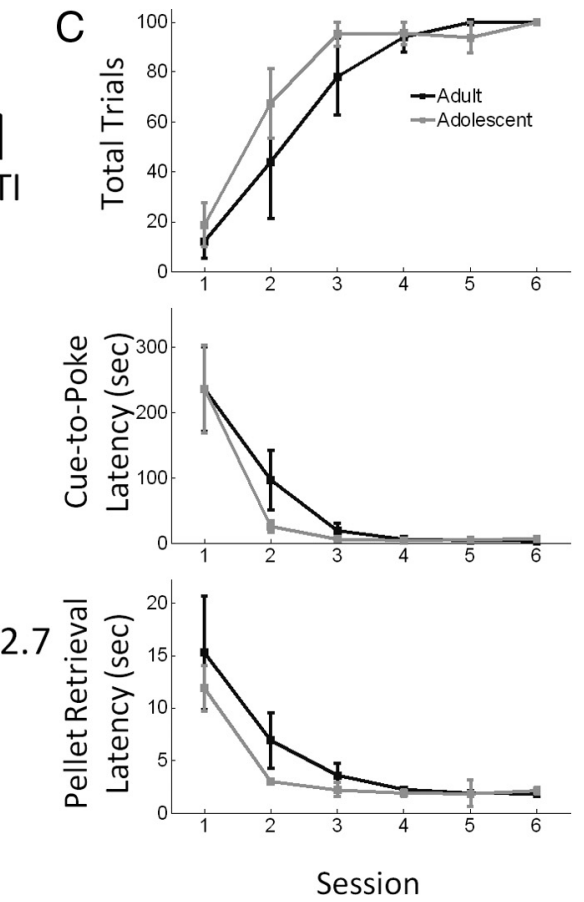

Session

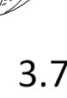

4.2

dult rats were trained on a simple instrumental learning paradigm in which they associated a nose poke (instrumental response) into a light-cued hole with the subsequent 列 task, with comparable between-age-group total trials (top), latencies from trial-onset cue to the instrumental response (middle), and latencies from instrumental response to food-pellet retrieval (bottom).

Table 1. Comparison of adolescent and adult unit activity in selected windows time-locked to task events

\begin{tabular}{|c|c|c|}
\hline Task event (Window) & Activated units & Inhibited units \\
\hline & Adult: 14/184 (7.6\%) & Adult: 23/184 (12.5\%) \\
\hline \multirow[t]{2}{*}{ Cue (0 to $1 \mathrm{~s}$ ) } & Adol: 11/265 (6.4\%) & Adol: $7 / 265$ (2.6\%) \\
\hline & Adult: 21/184 (11.4\%) & Adult: $34 / 184(18.5 \%)$ \\
\hline \multirow[t]{2}{*}{ Poke $(-0.5$ to $0 \mathrm{~s})$} & Adol: 19/265 (7.2\%) & Adol: 21/265 (7.9\%) \\
\hline & Adult: 25/184 (13.6\%) & Adult: 46/184 (25.0\%) \\
\hline \multirow[t]{2}{*}{ Poke $(0$ to $0.5 \mathrm{~s})$} & Adol: 32/265 (12.1\%) & Adol: 56/265 (21.1\%) \\
\hline & Adult: 28/184 (15.2\%) & Adult: $46 / 184(25.0 \%)$ \\
\hline \multirow[t]{2}{*}{ Poke (1 to $1.5 \mathrm{~s})$} & Adol: 51/265 (19.3\%) & Adol: $28 / 265(10.6 \%)$ \\
\hline & Adult: 35/184 (19.0\%) & Adult: 53/184 (28.8\%) \\
\hline \multirow[t]{2}{*}{ FT entry $(-0.5$ to $0 \mathrm{~s})$} & Adol: 80/265 (30.2\%) & Adol: 42/265 (15.9\%) \\
\hline & Adult: 34/184 (18.5\%) & Adult: 59/184 (32.1\%) \\
\hline \multirow[t]{2}{*}{ FT entry ( 0 to $0.5 \mathrm{~s}$ ) } & Adol: 79/265 (29.8\%) & Adol: 51/265 (19.3\%) \\
\hline & Adult: 31/184 (16.9\%) & Adult: 43/184 (23.4\%) \\
\hline FT entry (0.5 to $1 \mathrm{~s})$ & Adol: 38/265 (14.3\%) & Adol: 46/265 (17.4\%) \\
\hline
\end{tabular}

Windows of interest are time-locked to the cue, instrumental poke (Poke), or entry into the food trough (FT). The proportion of adolescent (Adol) and adult units that met criteria for significant activation or inhibition (see Materials and Methods) are indicated of the total number of units along with the categorized percentages in parentheses. In each case, significant $(p<0.05) \chi^{2}$ tests (that include number of activated, inhibited, and nonsignificant) units were followed up by direct age-related comparisons with Z tests of two proportions. Significant age-related proportional difference are indicated with bold type.

Electrophysiology analysis. Electrophysiological data were analyzed with custom-written scripts, executed in Matlab (MathWorks), along with the Chronux toolbox (http://chronux.org/) for LFP analyses and firing-rate variability functions graciously made available by Churchland 
A

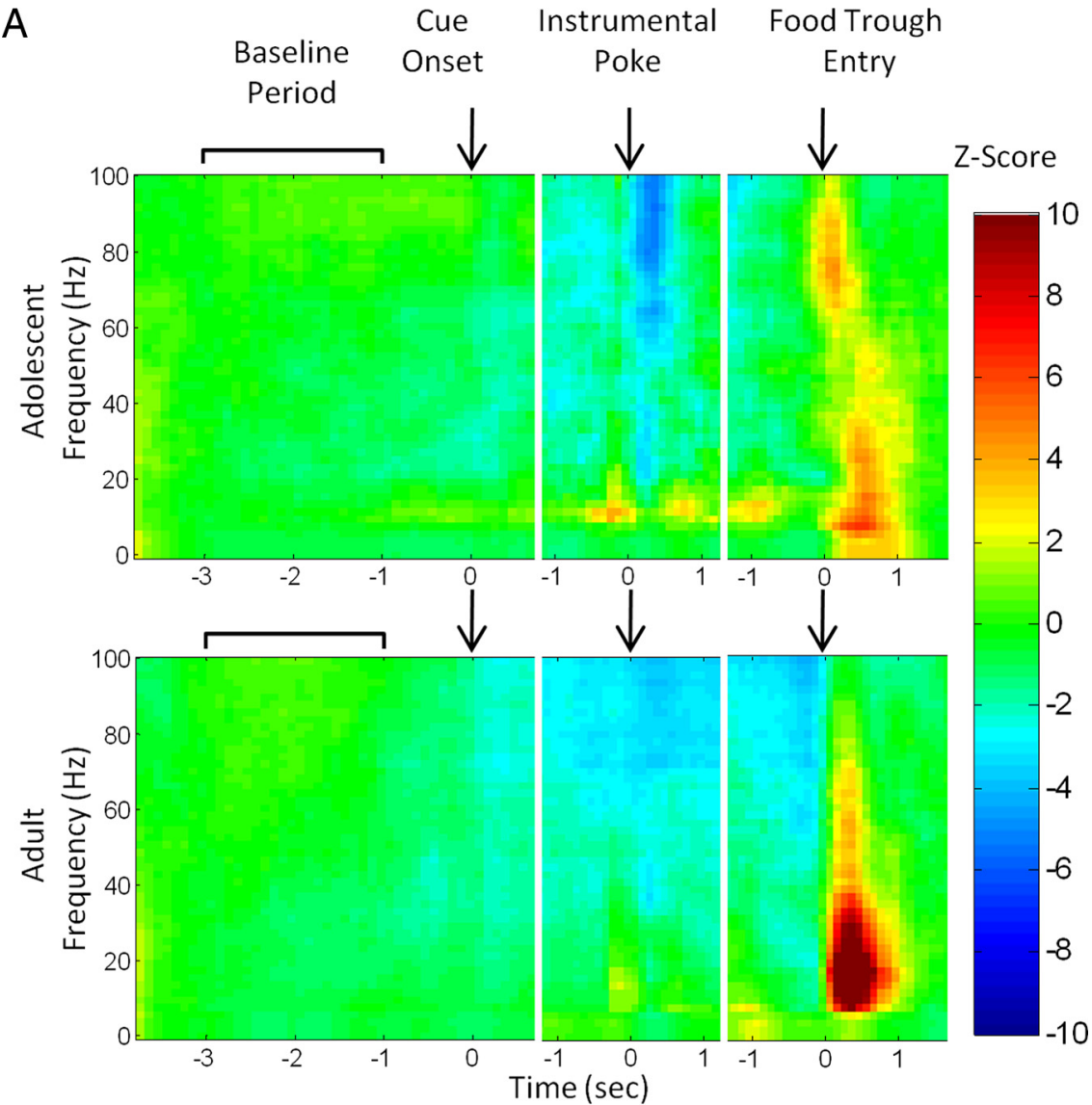

B
Theta
$(4-7 \mathrm{~Hz})$

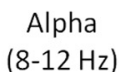
$(8-12 \mathrm{~Hz}$

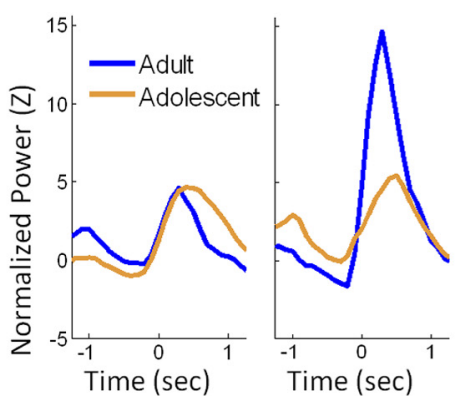

Low Gamma

$(31-75 \mathrm{~Hz})$

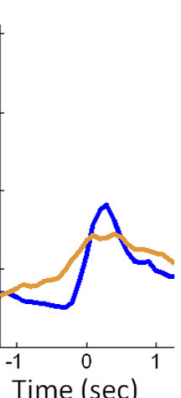

High Gamma

$(76-100 \mathrm{~Hz})$

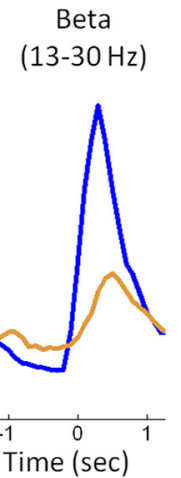

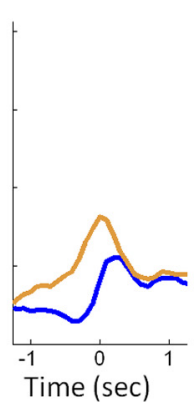

Figure 2. Adolescent and adult OFC LFPs during sessions 3-6. A, LFP power spectra for adolescents and adults in windows around key task events were normalized to the baseline period ( 3 to $1 \mathrm{~s}$ before cue onset) for each frequency. The time course of normalized LFP power was primarily similar between adolescents and adults. At cue onset, both groups exhibited slight reductions in gamma $(>30 \mathrm{~Hz}$ ) power. This was also observed around the instrumental response. Adults and adolescents both exhibited slightly increased beta $(13-30 \mathrm{~Hz})$ power at this time. Immediately after reinforcement, adolescents and adults had increases in theta $(4-7 \mathrm{~Hz})$, alpha $(8-12 \mathrm{~Hz})$, beta, and gamma power, with adults showing greater increases in the alpha and beta bands. Adolescents had greater increases in high gamma power (above $\sim 75 \mathrm{~Hz}$ ). $\boldsymbol{B}$, Time course of adolescent and adult normalized LFP power around reinforcement. Line graphs correspond to baseline-normalized adolescent and adult LFP power averaged across discrete frequency bands as labeled.

et al. (2010) (http://www.stanford.edu/ shenoy/GroupCodePacks. htm). In general, neural activity was time-locked to specific task events: trial-onset cue, instrumental nose-poke response, and food-trough entry. Raw LFP traces were time-locked to these task events, and trials with clipping artifacts were excluded before averaging. Example single-trial adolescent and adult raw LFP voltage traces during the period around reinforcement are presented (supplemental Fig. 1, available at www. jneurosci.org as supplemental material). Each subject's trial-averaged power spectrum in the several seconds around each task event was calculated by fast Fourier transform. This was done using 13 leading tapers, a time-bandwidth product of 7 , and a $1 \mathrm{~s}$ spanning moving window (in $250 \mathrm{~ms}$ steps). These parameters, compared with others that we examined, allowed for a frequency resolution of $\sim 2 \mathrm{~Hz}$, which generally allowed for multiple frequency bins in each band of interest. A multitaper approach was used because it improves spectrogram estimates when dealing with noninfinite time series data (Mitra and Pesaran, 1999), although using one, three, and nine tapers led to very similar spectrograms. Each frequency bin (row) in the power spectrum was $Z$-score normalized to the average spectral power during the baseline period (a $2 \mathrm{~s}$ window beginning $3 \mathrm{~s}$ before the cue). Normalized power spectra were averaged for adolescents and adults.

Perievent time firing-rate histograms were produced for each unit in windows around task events. The cross-trial average firing rate of each unit was $Z$-score normalized to that of its baseline period. Units were categorized as "activated" or "inhibited" within windows of interest based on whether their average normalized activity contained three consecutive 50 ms bins with $Z \geq 2$ or $Z \leq-2$, respectively. These criteria were validated using a nonparametric bootstrap analysis on the baseline period of each unit. For each unit, the baseline window was randomly sampled with replacement 10,000 times. The proportion of $2 \mathrm{~s}$ windows whose resampled activity reached significance criteria is a measure of the expected false-positive rate for that unit during any $2 \mathrm{~s}$ window. This led to an overall expected false-positive rate of $\alpha=0.0034$ for all adolescent units and $\alpha=0.0038$ for all adult units. These low $\alpha$ values indicate that unit false categorization would be rare enough as to not unduly impact statistical comparisons of category proportions between adolescents and adults. To compare the time course of unit responses, the categorization analysis was performed with a moving window around task events (movingwindow size of $500 \mathrm{~ms}$ in $250 \mathrm{~ms}$ steps). For time windows of particular interest for agerelated statistical comparisons (e.g., in the $1 \mathrm{~s}$ after the cue), $\chi^{2}$ analyses were performed, which included the number of adult and adolescent activated, inhibited, and nonsignificant units. Significant $\chi^{2}$ tests were followed by post hoc comparisons of proportions for each category (e.g., inhibited units between adolescents and adults) using a $Z$-test for two proportions (Table 1). Previous work with this behavioral paradigm has demonstrated that both adolescents and adults perform the instrumental response at a stable maximum by session 3 (Sturman et al., 2010). Therefore, except when otherwise noted, electrophysiological analyses are presented for sessions 3-6, at which point the action-outcome association is well learned by both groups. Here and elsewhere, the null hypothesis was rejected when $p<0.05$.

Analyses of firing-rate variability were calculated as Fano factors (spike count variance/mean) using an $80 \mathrm{~ms}$ moving window in $50 \mathrm{~ms}$ steps. For each unit, spike count variance and mean spike count were computed at each time point. The slope of the regression relating variance and mean for all units was determined at each window step, providing a Fano factor time course around task events. To examine whether observed changes in 
Fano factor over time (and age-related Fano factor differences) were attributable to changes in mean firing rate rather than variance, we performed a mean-matching technique devised by Churchland et al. (2010). In the first analysis, we performed mean matching separately for adolescent and adult units. This technique held the mean firing-rate distribution constant at each time point, by randomly and repeatedly discarding units. Fano factor estimates for each time point were based on the average of 10 iterations of this process. This procedure has been validated as an effective approach to avoiding artifacts attributable to firing-rate changes (Churchland et al., 2010). In addition to this, a separate mean-matching analysis was performed, in which the greatest common mean firing-rate histogram was used both across time within an age group (as above) and also between age groups. The observation of similar raw and mean-matched Fano factors would confirm that the time courses and age-related differences in Fano factor reflected spike-timing variability and were not merely artifacts of differences in mean firing-rate. Adolescent and adult Fano factors were statistically compared using rank-sum tests in Matlab.

\section{Results}

\section{Behavior}

During the behavioral task, adolescents poked into a light-cued hole to receive a food-pellet reinforcer (Fig. 1A). No significant differences were observed between adolescents and adults in the total number of trials $\left(F_{(1,1)}=1.3, p=0.28\right)$, latency from trial onset cue to the instrumental response $\left(F_{(1,1)}=0.34, p=0.57\right)$, or the latency from the instrumental response to food-pellet retrieval $\left(F_{(1,1)}=1.2, p=0.31\right)$. The task was consistently and maximally performed by adult and adolescent animals by the third training session (Fig. 1C).

\section{Local field potentials}

Electrophysiological recording of LFPs, a measure thought to reflect the activity of regional afferents, revealed somewhat similar patterns for adolescents and adults through much of the task, with notable differences in spectral power immediately after food-trough entry to receive reinforcement (Fig. $2 A$ ). At that time, adults exhibited greater alpha $(8-12 \mathrm{~Hz})$ and beta (13-30 $\mathrm{Hz})$ power. Theta $(4-7 \mathrm{~Hz})$ and low gamma $(31-75 \mathrm{~Hz})$ power were similar between groups, whereas adolescents had greater high gamma (76-100 Hz) power than adults (Fig. 2B).

\section{Fano factor analysis}

Age-related differences were observed in firing-rate variability associated with specific task events. The Fano factor, which is the slope of the relationship between spike-count variance and spikecount mean (Churchland et al. 2010), was computed to examine the variability of spike timing across trials (Fig. 3). Adolescents (8 rats, 265 units) had significantly larger Fano factors than adults (4 rats, 184 units) during sessions 3-6 (comparisons performed with rank-sum tests) during the 2 s baseline period $(Z=6.90, p<$ $0.01)$ in a $1 \mathrm{~s}$ window immediately after the trial-onset cue $(Z=$ $5.48, p<0.01)$ in a $1 \mathrm{~s}$ window centered around the instrumental response $(Z=3.12, p<0.01)$ and in the one second leading up to reinforcement retrieval ( $Z=3.77, p<0.01$ ) (Fig. 3). Because
Fano factor calculations depend on window size and step, we varied these parameters in the period around the instrumental poke to demonstrate that, although the magnitude and smoothness of the calculations are affected, the general time course and age-related differences remain (supplemental Fig. 2, available at www.jneurosci.org as supplemental material). We performed a mean-matching technique (Churchland et al., 2010) to hold the mean firing rate approximately constant over time so that temporal firing-rate changes would not obscure our interpretation of the Fano factor as a measure of variability (supplemental Fig. $3 A$, available at www.jneurosci.org as supplemental material). We similarly equalized firing-rate distributions between age groups (supplemental Fig. 3b, available at www.jneurosci.org as supplemental material). Raw Fano factors were very similar to those computed with either mean-matching method, confirming that the observed Fano factor time course reflects the variability in spike timing regardless of mean firing-rate dynamics. One exception to this was after reinforcement retrieval, at which time adults exhibited greater raw Fano factors (Fig. 3). This difference was attributable, at least in part, to changes in mean firing rate, because there was no statistically significant difference in the meanmatched Fano factors during that period (supplemental Fig. 3, available at www.jneurosci.org as supplemental material). These findings indicate that salient events lead to a reduction in the variability of spike timing for both adolescents and adults and that, interestingly, adolescent OFC neural spike timing is generally more variable than that of adults throughout much of the task. Stimulus-driven Fano factor reductions are thought to be a general property of cortical architecture (Churchland et al. 2010). Thus, higher Fano factors may suggest an intrinsic tendency for spike timing to be less tightly controlled in the OFC of adolescents compared with adults.

\section{Unit activity}

Analysis of single-unit neural activity during the task revealed substantial event-specific differences between adolescents and adults. During session 1, before learning the action-outcome 


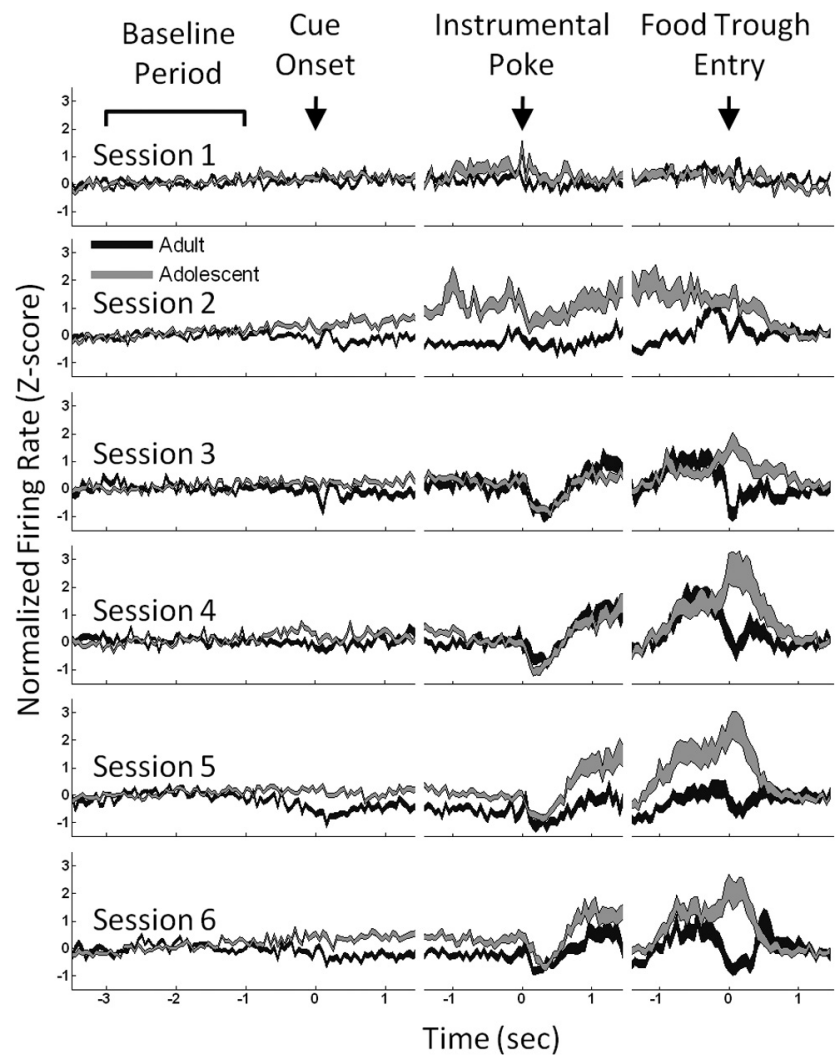

Figure 4. Average baseline-normalized firing rate +1 SEM (shading) for all adult and adolescent units, time-locked to task events during each of six sessions. The median taskwide firing rate for all adolescent units was $4.66 \mathrm{~Hz}$, and all adult units was $5.18 \mathrm{~Hz}$. Although slight, their corresponding firing-rate distributions were significantly different (rank-sum test, $Z=$ 2.18, $p=0.03$ ). This figure demonstrates that, in session 1 (adult, $n=47$; adolescent, $n=60$; first row), when the action- outcome association was not yet learned (Fig. 1c), there was little task-related activity to the cue (left), instrumental pokes (middle), or rewarded food-trough entries (right) in either group. By session 2 (adult, $n=59$; adolescent, $n=60$; second row), as animals learned at different rates and performed the task to varying extents, average $0 \mathrm{FC}$ neural activity began to change around task events in both groups. From session 3 onward (session 3 : adult, $n=49$; adolescent, $n=64$; session 4: adult, $n=46$; adolescent, $n=67$; session 5 : adult, $n=41$; adolescent, $n=72$; session 6: adult, $n=48$; adolescent, $n=62$; third to sixth rows), average normalized neural activity settled into somewhat stable patterns in both age groups.

associations, unit activity changed little to task events in either group. Once the task was well learned (training sessions 3-6), however, task events elicited consistent patterns of neural activity (Fig. 4). The baseline-normalized firing rates of each unit timelocked to task events are shown in Figure $5 A$, illustrating the range and extent of phasic neural activity. In adults (4 rats, 184 units), but not adolescents ( 8 rats, 265 units), average activity was reduced at the cue and preceding the instrumental response (Fig. $5 B$ ). After the response, the normalized population activity of both groups similarly dropped, with adolescents rebounding more than adults. Around the time of reinforcement, population activity increased, with adults peaking earlier and at a lower level than adolescents. Maximal adolescent activity was reached at the time of food-trough entry, at which point average adult activity was far lower. Although too few in number to draw a strong conclusion, adolescent ( $n=8$ units) and adult ( $n=5$ units) putative fast-spiking (FS) interneurons exhibited a similar general pattern of activity around events of interest as the general population of units during sessions 3-6 (supplemental Fig. 4, available at www.jneurosci.org as supplemental material). Com- parisons of the proportion of excitatory and inhibitory phasic activity to task events (Fig. $5 \mathrm{C}$ ) generally revealed reduced inhibitory responses and similar or enhanced excitatory responses in adolescents. In the $1 \mathrm{~s}$ after the cue, adults had a significantly larger proportion of inhibited units than adolescents with comparable proportions of activated units (Table 1). After the instrumental response, when adolescents and adults had similar reductions in population activity, similar proportions of activated and inhibited units were observed. A moving-window categorization analysis, used to visualize the time course of neural recruitment, demonstrated that, around the instrumental response, adult inhibited units became inhibited earlier and were sustained longer than in adolescents (Fig. 5C). This is confirmed by examining proportions of inhibited units in time windows $0.5 \mathrm{~s}$ before and $1-1.5 \mathrm{~s}$ after the instrumental response (Table 1 ). Although adult activated units also appear to be recruited before those of adolescents, these differences were not statistically significant. The proportions of units categorized as activated and inhibited differed substantially around reinforcement, with adults having greater proportions of inhibited units and adolescents having greater proportions of activated units. By $0.5-1 \mathrm{~s}$ after reinforcement, there were no age-related differences in unit categorization. These findings demonstrate that, although similar proportions of adolescent and adult units can become activated or inhibited at different times (e.g., instrumental poke), through much of the task adolescents had smaller proportions of inhibited units.

\section{Discussion}

At both population and single-unit levels, the adolescent OFC processed reward-motivated behavior differently from that of adults, with the most prominent distinction being less pronounced adolescent reductions in neural activity during reward and other salient events. Adolescents also exhibited greater crosstrial spike-timing variability throughout much of the task. During reinforcement, in addition to less reductions in activity, there was a larger proportion of adolescent units that increased their activity, as well as differences in alpha, beta, and gamma LFP power compared with adults. It is important that these agerelated neural processing differences were observed although task performance was similar, which indicates that such differences do not simply reflect a behavioral confound (Schlaggar et al., 2002; Yurgelun-Todd, 2007). Even if adding additional subjects were to reveal behavioral differences during early training, both adolescents and adults performed the task at a maximal level from the third session onward. Our electrophysiology analyses focused on these later sessions, when the action-outcome association was well learned by both groups. We chose a behavioral task that, although simple enough to be learned in the short timeframe of rat adolescence, could be considered a basic building block of more complex motivated behavior. Thus, these findings indicate that, even as adolescents perform the same motivated behavior as adults, their neural encoding of salient events and apparent processing efficiency (as it relates to spike-timing variability) fundamentally differ.

Adolescent neurons tended to have less reduced activity than adults during important behavioral events such as the trial-onset cue, before the instrumental response, and before and during reward. Such age-related differences could be attributable to less OFC neuronal inhibition at these times. Neuronal inhibition plays a critical role in synchronizing oscillatory activity (Fries et al., 2007; Cardin et al., 2009; Sohal et al., 2009), controlling precise spike timing, and improving the efficiency of neuronal com- 
munication (Buzsáki and Chrobak, 1995). Such oscillations, as measured with EEG and LFP, are rhythmic fluctuations in neuronal excitability, thought to reflect the interactions of intrinsic cellular and circuit properties (Buzsáki and Draguhn, 2004), which fine-tune the timing of spike output (Fries, 2005). Synchrony of oscillations may provide a conduit for the communication of neuronal groups (Fries, 2005) and may be central to perceptual binding and other processes (Uhlhaas et al., 2009b). Measures of neuronal synchrony in specific frequency bands correlate with cognitive performance in numerous contexts (Başar et al., 2000) and are reduced in several pathological states, such as schizophrenia (Uhlhaas and Singer, 2010). Uhlhaas et al. (2009a) found differences in task-related EEG oscillations between human adolescents and adults. Consistent with these findings, we observed smaller increases in alpha and beta power in the OFC of adolescents during reinforcement. These frequency bands are thought to be important for neural communication over longer distances (Pfurtscheller et al., 2000; Brovelli et al., 2004; Klimesch et al., 2007), which could be less efficient in adolescents. This interpretation is consistent with studies showing that functional connectivity changes from being more local to more distributed through development (Fair et al., 2009; Somerville and Casey, 2010).

We also observed age-related differences in firing-rate variability across trials, assessed using a Fano factor analysis. Recent work has demonstrated that, in many cortical regions, neuron spiking activity is stabilized by stimuli or instrumental behavior, as reflected in reduced Fano factors (Churchland et al., 2010). Indeed, we observed that, in the OFC, instrumental behavior, reward approach/anticipation, and reinforcement (in adults) led to reductions in our measure of firing-rate variability. The largest reductions in variability occurred as rats performed the instrumental response and in the period before reinforcement. Greater firing-rate variability would be expected if the timing of phasic neural activity was less tightly controlled, as may be the case in the OFC of adolescents. Adolescents had greater Fano factors than adults through much of the task, with the exception of the $1 \mathrm{~s}$ period immediately after foodtrough entry. These results indicate that adolescents tend to have greater firing-rate variability, which may suggest reduced efficiency in neural coding. That is, greater Fano factors indicates that adolescent OFC neurons encode the same salient events with more variability, from trial to trial, which could in turn mean lower signal-to-noise ratios in the corresponding rate code compared with that of adults. This is consistent with the finding that the event-related potentials of children and adolescents have lower signal-to-noise ratios than adults, which could be attribut-

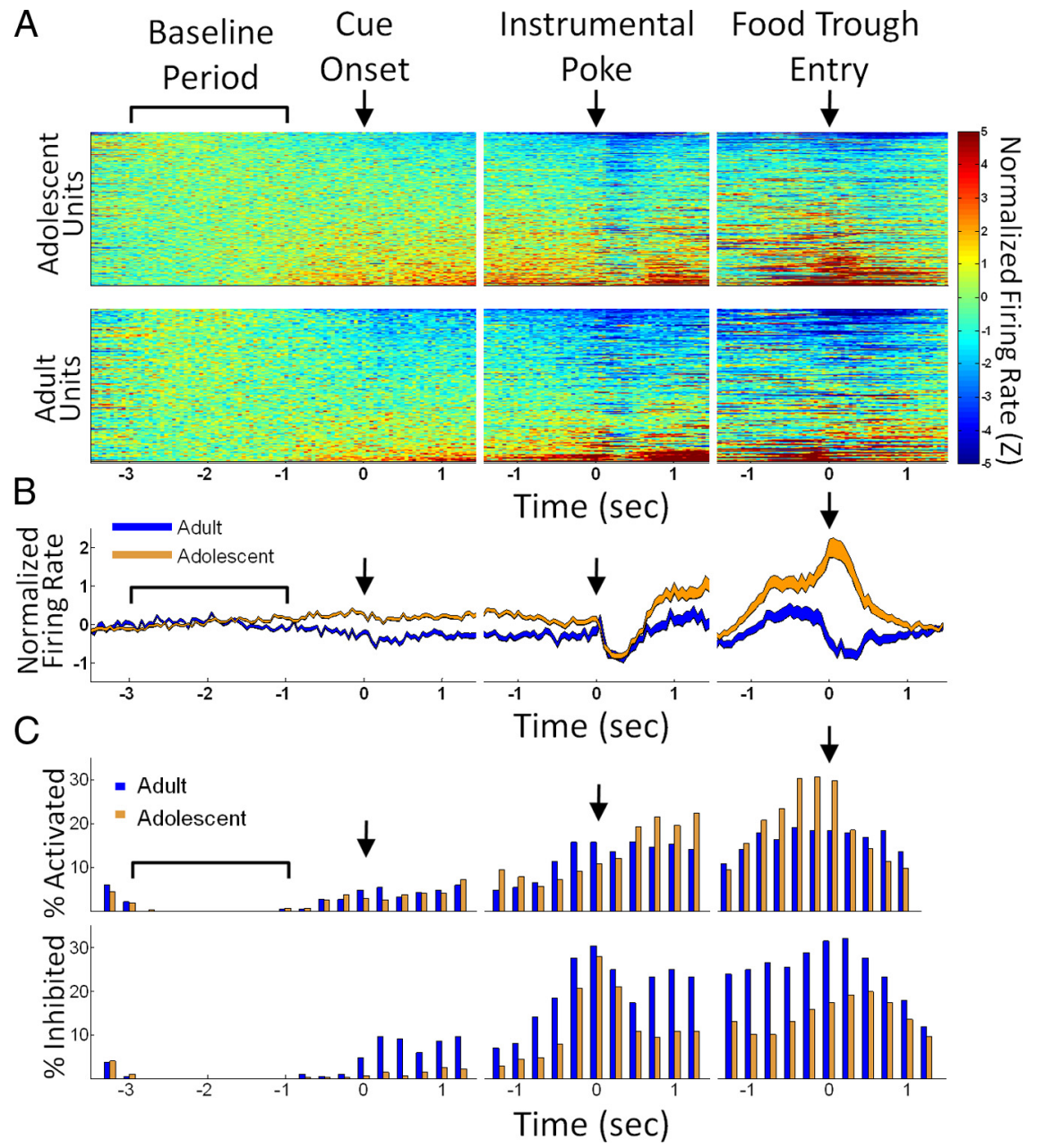

Figure 5. Phasic $0 \mathrm{FC}$ population and single-unit activity during sessions 3-6. A, Heat plots represent the baseline-normalized firing rate for each adolescent ( $n=265$; top plots) and adult ( $n=184$; bottom plots) unit. Each row is the activity of an individual unit in $50 \mathrm{~ms}$ time bins aligned to corresponding events of interest and sorted from lowest to highest average normalized firing . Arrows indicate the timing of task events. $\boldsymbol{B}$, Average normalized firing rate (across all units) +1 SEM (shading) for adults , during, and after cue onset. This continues until animals perform the instrumental response (middle). At that time, average unit intere categorized as activated (top) or inhibited (bottom) are identified over time using a moving-window analysis 列 sustained manner. Around the time of reinforcement (right), adolescents ultimately had higher percentages of activated units, whereas adults consistently had higher percentages of inhibited units.

able to "intra-individual instability" of brain regions producing these signals (Segalowitz et al., 2010). Just as neural inhibition is critical for entraining oscillations, inhibitory networks provide precision timing for the spiking of principal cells (Buzsáki and Chrobak, 1995). Thus, a connection may exist between the tendency for adolescent units to exhibit less phasic inhibition to salient events and the greater firing-rate variability of adolescent units. We must express caution, however, that such a connection is not likely direct, because the timing of the greatest Fano factor disparities was not also the timing of largest differences in phasic inhibition.

Vast neurodevelopmental changes occur during adolescence. There is a reduction in gray matter and augmentation of white matter during this period (Benes et al., 1994; Paus et al., 1999, 
2001; Sowell et al., 2001, 2002, 2003; Gogtay et al., 2004). Receptors for several neuromodulators such as dopamine are expressed at higher levels in adolescents than in adults in PFC and basal ganglia (Gelbard et al., 1989; Lidow and Rakic, 1992; Teicher et al., 1995; Tarazi et al., 1999; Tarazi and Baldessarini, 2000). In anesthetized rats, the spontaneous neural activity of dopamine neurons is greater in adolescents than juveniles or adults (McCutcheon and Marinelli, 2009). In cortical slices, activating effects of a dopamine $\mathrm{D}_{2}$ receptor agonist are only present by late adolescence or early adulthood, at which time a sudden shift is observed (Tseng and O'Donnell, 2007). The expression of NMDA receptors on FS neurons also changes dramatically in the PFC of adolescents. The majority of adolescent FS interneurons exhibit no synaptic NMDA receptor-mediated currents. Those cells that do have them exhibit a far-reduced NMDA/AMPA ratio (Wang and Gao, 2009). These studies demonstrate fundamental differences in the architecture and physiology of adolescent brain regions and transmitters associated with both normal motivated behavior and mental illnesses. The present study, which to our knowledge is the first to use extracellular electrophysiological recording in awake, behaving adolescent animals, advances the functional relevance of these cellular and molecular findings by demonstrating that task-related neural activity is fundamentally different in adolescents during the processing of salient events.

Human fMRI studies have found that adolescents process reward and reward-anticipation differently than adults at a largerscale regional level (Ernst et al., 2005; Galvan et al., 2006; Geier et al., 2010; Van Leijenhorst et al., 2010). Current explanations for some adolescent behavioral vulnerabilities include the notion that the PFC is "underdeveloped" in terms of its activity and/or its functional connectivity with and modulation of subcortical structures (Ernst et al., 2006; Casey et al., 2008; Steinberg, 2008). The present study finds that developmental differences are observable even during very basic reward-motivated behavior and are fundamentally manifested at the single-unit level by a reduced propensity for reduced neural activity in adolescent OFC to most, but not all, salient events. Although future work is needed to establish such a connection, differences at the single-unit level in the proportions of inhibitory responses may be the source of some of the adolescent differences observed in oscillatory power and spike-timing variability. Because of the importance of inhibition in entraining oscillations, controlling the precise timing of spikes, and thus facilitating efficient communication of neuronal groups (Buzsáki and Chrobak, 1995; Fries et al., 2007), reduced adolescent PFC inhibition is consistent with the observation of large-scale differences in cortical processing seen in this study and others. However, the tendency for adolescents to have less reductions in unit activity around salient events may result from lower reductions in the activity of excitatory afferents as well as reduced inhibition.

Altered cortical inhibitory activity may influence behavioral inhibition (Chudasama et al., 2003; Narayanan and Laubach, 2006) and has been associated with some pathological states (Chamberlain et al., 2005; Lewis et al., 2005; Behrens and Sejnowski, 2009; Lewis, 2009). For example, individuals with schizophrenia have reduced GAD67 mRNA expression, an enzyme involved in the synthesis of the inhibitory neurotransmitter GABA (Akbarian et al., 1995). Schizophrenia patients also have reduced GABA membrane transporter (GAT-1)-immunoreactive axon cartridges in the PFC (Woo et al., 1998). This is of particular relevance to research in adolescents, because GAT-1-immunoreactive cartridges (which are also immunoreactive to parvalbumin) peak just before adolescence and then undergo a dramatic reduction through late adolescence (Cruz et al.,
2003), the typical onset time for schizophrenia. Future work delineating the precise source of age-related phasic activity differences during normal development may be directly relevant to the pathophysiology and symptomatic time course of psychiatric illnesses that arise during adolescence.

\section{References}

Adriani W, Granstrem O, Macri S, Izykenova G, Dambinova S, Laviola G (2004) Behavioral and neurochemical vulnerability during adolescence in mice: studies with nicotine. Neuropsychopharmacology 29:869-878.

Akbarian S, Kim JJ, Potkin SG, Hagman JO, Tafazzoli A, Bunney WE Jr, Jones EG (1995) Gene expression for glutamic acid decarboxylase is reduced without loss of neurons in prefrontal cortex of schizophrenics. Arch Gen Psychiatry 52:258-266.

Arnett JJ (1999) Adolescent storm and stress, reconsidered. Am Psychol 54:317-326.

Başar E, Başar-Eroğlu C, Karakaş S, Schürmann M (2000) Brain oscillations in perception and memory. Int J Psychophysiol 35:95-124.

Behrens MM, Sejnowski TJ (2009) Does schizophrenia arise from oxidative dysregulation of parvalbumin-interneurons in the developing cortex? Neuropharmacology 57:193-200.

Benes FM, Turtle M, Khan Y, Farol P (1994) Myelination of a key relay zone in the hippocampal formation occurs in the human brain during childhood, adolescence, and adulthood. Arch Gen Psychiatry 51:477-484.

Brenhouse HC, Sonntag KC, Andersen SL (2008) Transient D dopamine receptor expression on prefrontal cortex projection neurons: relationship to enhanced motivational salience of drug cues in adolescence. J Neurosci 28:2375-2382.

Brovelli A, Ding M, Ledberg A, Chen Y, Nakamura R, Bressler SL (2004) Beta oscillations in a large-scale sensorimotor cortical network: directional influences revealed by Granger causality. Proc Natl Acad Sci U S A 101:9849-9854.

Buzsáki G, Chrobak JJ (1995) Temporal structure in spatially organized neuronal ensembles: a role for interneuronal networks. Curr Opin Neurobiol 5:504-510.

Buzsáki G, Draguhn A (2004) Neuronal oscillations in cortical networks. Science 304:1926-1929.

Cardin JA, Carlén M, Meletis K, Knoblich U, Zhang F, Deisseroth K, Tsai LH, Moore CI (2009) Driving fast-spiking cells induces gamma rhythm and controls sensory responses. Nature 459:663-667.

Casey BJ, Getz S, Galvan A (2008) The adolescent brain. Dev Rev 28:62-77.

Cauffman E, Shulman EP, Steinberg L, Claus E, Banich MT, Graham S, Woolard J (2010) Age differences in affective decision making as indexed by performance on the Iowa Gambling Task. Dev Psychol 46:193-207.

Chamberlain SR, Blackwell AD, Fineberg NA, Robbins TW, Sahakian BJ (2005) The neuropsychology of obsessive compulsive disorder: the importance of failures in cognitive and behavioural inhibition as candidate endophenotypic markers. Neurosci Biobehav Rev 29:399-419.

Chudasama Y, Passetti F, Rhodes SE, Lopian D, Desai A, Robbins TW (2003) Dissociable aspects of performance on the 5-choice serial reaction time task following lesions of the dorsal anterior cingulate, infralimbic and orbitofrontal cortex in the rat: differential effects on selectivity, impulsivity and compulsivity. Behav Brain Res 146:105-119.

Churchland MM, Yu BM, Cunningham JP, Sugrue LP, Cohen MR, Corrado GS, Newsome WT, Clark AM, Hosseini P, Scott BB, Bradley DC, Smith MA, Kohn A, Movshon JA, Armstrong KM, Moore T, Chang SW, Snyder LH, Lisberger SG, Priebe NJ, Finn IM, Ferster D, Ryu SI, Santhanam G, Sahani M, Shenoy KV (2010) Stimulus onset quenches neural variability: a widespread cortical phenomenon. Nat Neurosci 13:369-378.

Cruz DA, Eggan SM, Lewis DA (2003) Postnatal development of pre- and postsynaptic GABA markers at chandelier cell connections with pyramidal neurons in monkey prefrontal cortex. J Comp Neurol 465:385-400.

Ernst M, Nelson EE, Jazbec S, McClure EB, Monk CS, Leibenluft E, Blair J, Pine DS (2005) Amygdala and nucleus accumbens in responses to receipt and omission of gains in adults and adolescents. Neuroimage 25:1279-1291.

Ernst M, Pine DS, Hardin M (2006) Triadic model of the neurobiology of motivated behavior in adolescence. Psychol Med 36:299-312.

Fair DA, Cohen AL, Power JD, Dosenbach NU, Church JA, Miezin FM, Schlaggar BL, Petersen SE (2009) Functional brain networks develop from a "local to distributed" organization. PLoS Comput Biol 5:e1000381.

Figner B, Mackinlay RJ, Wilkening F, Weber EU (2009) Affective and delib- 
erative processes in risky choice: age differences in risk taking in the Columbia Card Task. J Exp Psychol Learn Mem Cogn 35:709-730.

Friemel CM, Spanagel R, Schneider M (2010) Reward sensitivity for a palatable food reward peaks during pubertal developmental in rats. Front Behav Neurosci 4:39.

Fries P (2005) A mechanism for cognitive dynamics: neuronal communication through neuronal coherence. Trends Cogn Sci 9:474-480.

Fries P, Nikolić D, Singer W (2007) The gamma cycle. Trends Neurosci 30:309-316.

Galvan A, Hare TA, Parra CE, Penn J, Voss H, Glover G, Casey BJ (2006) Earlier development of the accumbens relative to orbitofrontal cortex might underlie risk-taking behavior in adolescents. J Neurosci 26:6885-6892.

Geier CF, Terwilliger R, Teslovich T, Velanova K, Luna B (2010) Immaturities in reward processing and its influence on inhibitory control in adolescence. Cereb Cortex 20:1613-1629.

Gelbard HA, Teicher MH, Faedda G, Baldessarini RJ (1989) Postnatal development of dopamine D1 and D2 receptor sites in rat striatum. Brain Res Dev Brain Res 49:123-130.

Gogtay N, Giedd JN, Lusk L, Hayashi KM, Greenstein D, Vaituzis AC, Nugent TF 3rd, Herman DH, Clasen LS, Toga AW, Rapoport JL, Thompson PM (2004) Dynamic mapping of human cortical development during childhood through early adulthood. Proc Natl Acad Sci U S A 101:8174-8179.

Homayoun H, Moghaddam B (2008) Orbitofrontal cortex neurons as a common target for classic and glutamatergic antipsychotic drugs. Proc Natl Acad Sci U S A 105:18041-18046.

Johnston L, O'Malley P, Bachman J, Schulenberg J (2008) Monitoring the future: national survey results on adolescent drug use: overview of key findings. Bethesda, MD: National Institutes of Health.

Klimesch W, Sauseng P, Hanslmayr S (2007) EEG alpha oscillations: the inhibition-timing hypothesis. Brain Res Rev 53:63-88.

Lewis DA (2009) Neuroplasticity of excitatory and inhibitory cortical circuits in schizophrenia. Dialogues Clin Neurosci 11:269-280.

Lewis DA, Hashimoto T, Volk DW (2005) Cortical inhibitory neurons and schizophrenia. Nat Rev Neurosci 6:312-324.

Lidow MS, Rakic P (1992) Scheduling of monoaminergic neurotransmitter receptor expression in the primate neocortex during postnatal development. Cereb Cortex 2:401-416.

Liston C, Watts R, Tottenham N, Davidson MC, Niogi S, Ulug AM, Casey BJ (2006) Frontostriatal microstructure modulates efficient recruitment of cognitive control. Cereb Cortex 16:553-560.

McCutcheon JE, Marinelli M (2009) Age matters. Eur J Neurosci 29:997-1014.

Mitra PP, Pesaran B (1999) Analysis of dynamic brain imaging data. Biophys J 76:691-708.

Narayanan NS, Laubach M (2006) Top-down control of motor cortex ensembles by dorsomedial prefrontal cortex. Neuron 52:921-931.

Paus T (2010) Growth of white matter in the adolescent brain: myelin or axon? Brain Cogn 72:26-35.

Paus T, Zijdenbos A, Worsley K, Collins DL, Blumenthal J, Giedd JN, Rapoport JL, Evans AC (1999) Structural maturation of neural pathways in children and adolescents: in vivo study. Science 283:1908-1911.

Paus T, Collins DL, Evans AC, Leonard G, Pike B, Zijdenbos A (2001) Maturation of white matter in the human brain: a review of magnetic resonance studies. Brain Res Bull 54:255-266.

Paxinos G, Watson C (1998) The rat brain in stereotaxic coordinates, Ed 4. San Diego: Academic.

Pfurtscheller G, Neuper C, Pichler-Zalaudek K, Edlinger G, Lopes da Silva FH (2000) Do brain oscillations of different frequencies indicate interaction between cortical areas in humans? Neurosci Lett 286:66-68.

Pine DS (2002) Brain development and the onset of mood disorders. Semin Clin Neuropsychiatry 7:223-233.

Schlaggar BL, Brown TT, Lugar HM, Visscher KM, Miezin FM, Petersen SE (2002) Functional neuroanatomical differences between adults and schoolage children in the processing of single words. Science 296:1476-1479.

Schoenbaum G, Roesch MR, Stalnaker TA, Takahashi YK (2009) A new perspective on the role of the orbitofrontal cortex in adaptive behaviour. Nat Rev Neurosci 10:885-892.

Schultz W, Tremblay L, Hollerman JR (2000) Reward processing in primate orbitofrontal cortex and basal ganglia. Cereb Cortex 10:272-284.

Segalowitz SJ, Santesso DL, Jetha MK (2010) Electrophysiological changes during adolescence: a review. Brain Cogn 72:86-100.

Sohal VS, Zhang F, Yizhar O, Deisseroth K (2009) Parvalbumin neurons and gamma rhythms enhance cortical circuit performance. Nature 459:698-702.

Somerville LH, Casey BJ (2010) Developmental neurobiology of cognitive control and motivational systems. Curr Opin Neurobiol 20:236-241.

Sowell ER, Thompson PM, Tessner KD, Toga AW (2001) Mapping continued brain growth and gray matter density reduction in dorsal frontal cortex: inverse relationships during postadolescent brain maturation. J Neurosci 21:8819-8829.

Sowell ER, Trauner DA, Gamst A, Jernigan TL (2002) Development of cortical and subcortical brain structures in childhood and adolescence: a structural MRI study. Dev Med Child Neurol 44:4-16.

Sowell ER, Peterson BS, Thompson PM, Welcome SE, Henkenius AL, Toga AW (2003) Mapping cortical change across the human life span. Nat Neurosci 6:309-315.

Spear LP (2000) The adolescent brain and age-related behavioral manifestations. Neurosci Biobehav Rev 24:417-463.

Spear LP, Brake SC (1983) Periadolescence: age-dependent behavior and psychopharmacological responsivity in rats. Dev Psychobiol 16:83-109.

Steinberg L (2008) A social neuroscience perspective on adolescent risktaking. Dev Rev 28:78-106.

Sturman DA, Mandell DR, Moghaddam B (2010) Adolescents exhibit behavioral differences from adults during instrumental learning and extinction. Behav Neurosci 124:16-25.

Tarazi FI, Baldessarini RJ (2000) Comparative postnatal development of dopamine $\mathrm{D}_{1}, \mathrm{D}_{2}$ and $\mathrm{D}_{4}$ receptors in rat forebrain. Int J Dev Neurosci 18:29-37.

Tarazi FI, Tomasini EC, Baldessarini RJ (1999) Postnatal development of dopamine D1-like receptors in rat cortical and striatolimbic brain regions: an autoradiographic study. Dev Neurosci 21:43-49.

Teicher MH, Andersen SL, Hostetter JC Jr (1995) Evidence for dopamine receptor pruning between adolescence and adulthood in striatum but not nucleus accumbens. Brain Res Dev Brain Res 89:167-172.

Totah NK, Kim YB, Homayoun H, Moghaddam B (2009) Anterior cingulate neurons represent errors and preparatory attention within the same behavioral sequence. J Neurosci 29:6418-6426.

Tseng KY, O'Donnell P (2007) Dopamine modulation of prefrontal cortical interneurons changes during adolescence. Cereb Cortex 17:1235-1240.

Uhlhaas PJ, Singer W (2010) Abnormal neural oscillations and synchrony in schizophrenia. Nat Rev Neurosci 11:100-113.

Uhlhaas PJ, Roux F, Singer W, Haenschel C, Sireteanu R, Rodriguez E (2009a) The development of neural synchrony reflects late maturation and restructuring of functional networks in humans. Proc Natl Acad Sci U S A 106:9866-9871.

Uhlhaas PJ, Pipa G, Lima B, Melloni L, Neuenschwander S, Nikolić D, Singer W (2009b) Neural synchrony in cortical networks: history, concept and current status. Front Integr Neurosci 3:17.

Van Leijenhorst L, Zanolie K, Van Meel CS, Westenberg PM, Rombouts SA, Crone EA (2010) What motivates the adolescent? Brain regions mediating reward sensitivity across adolescence. Cereb Cortex 20:61-69.

Volkmar FR (1996) Childhood and adolescent psychosis: a review of the past 10 years. J Am Acad Child Adolesc Psychiatry 35:843-851.

Wang HX, Gao WJ (2009) Cell type-specific development of NMDA receptors in the interneurons of rat prefrontal cortex. Neuropsychopharmacology 34:2028-2040.

Woo TU, Whitehead RE, Melchitzky DS, Lewis DA (1998) A subclass of prefrontal gamma-aminobutyric acid axon terminals are selectively altered in schizophrenia. Proc Natl Acad Sci U S A 95:5341-5346.

Yurgelun-Todd D (2007) Emotional and cognitive changes during adolescence. Curr Opin Neurobiol 17:251-257. 